\title{
Topography and morphometry of intestinal mast cells in children with Hirschsprug's disease.
}

\author{
Adam Hermanowicz ${ }^{1}$, Wojciech Dębek ${ }^{1}$, Ewa Dzienis-Koronkiewicz ${ }^{1}$, \\ Lech Chyczewski
}

${ }^{1}$ Department of Pediatric Surgery, ${ }^{2}$ Department of Clinical Molecular Biology, Medical University, Białystok, Poland

\begin{abstract}
Mast cells (MC) are source of many biological active compounds like cytokines, arachidonic acid derivates, proteoglicanes, prostaglandins, proteases, free oxygen radials, NGF, PAF and many more. The role of MC in pathogenesis of Hirschsprung's disease (HD) is not clear. Substances produced by MC may exert an important effect on embryology, growth, differentiation and regeneration of intestinal nervous system. Additionally, MC products modulate inflammation processes thus influencing on the clinical course of HD. Present study was established to evaluate the morphologic MC examination as a support of making diagnosis in HD. The MC topography and morphometry were evaluated in specimens collected from aganglionic colon of patients with diagnosed HD. The results were compared with corresponding data from normally innervated colon of patients suffering from constipation, and normal colon of children not presenting defecation problems. $\mathrm{MC}$ were visualized using indirect immunohistochemical method LSAB with mouse antibody against human tryptase. The MC visualized in submucosa and muscular layer in Hirschsprung's disease were significantly larger in comparison with control group ( $\mathrm{p}<0.05$ ). Also the number of $\mathrm{MC} / \mathrm{mm}^{2}$ in mucosa and lamina propria in $\mathrm{HD}$ was significantly elevated ( $<<0.05$ ). However, the MC density in submucosa was also higher but it was not high statistically significant. In muscular layer and in serosa density of $\mathrm{MC} / \mathrm{mm}^{2}$ was not statistically significant. In the intestinal wall $\mathrm{MC}$ in aganglionic segment in Hirschsprung's disease are significantly activated comparing with normally innervated colon segments taken from the patients from other groups. This may confirm the role of MC both in pathogenesis of HD and in the reparation processes of bowel nervous system.
\end{abstract}

Key words: Hirschsprung disease - Mast cell - Morphometry - Enteric nervous system

\section{Introduction}

Hirschsprung's disease (HD) is rare (1/5000 newborns) but potentially severe illness resulting from the bowel nervous system dysfunction. Symptoms of HD like delayed meconium passage in newborns, intestinal obstruction, constipation toxic megacolon, as well as failure to thrive, are consequences of absence or hypothrophy of intramural nervous ganglia. The aganglionic colon wall segment contains instead increased number of hypertrophied parasympathetic nerve trunks. Although intensively studied, the details of multifactorial pathogenesis of HD remain not clear [1-4].

Correspondence: W. Dębek, Dept. of Pediatric Surgery, Medical University, Waszyngtona Str. 17, 15-274 Białystok, Poland; tel.: (+4885) 7450920, fax.: (+4885) 7450921, e-mail:wdebek@hotmail.com
Mast cells (MC) are known to be involved in many functions in human body. They are observed in great amount in contact with blood vessels and nerve fibers [5-10,23]. Among a great variety of biological active substances, MC synthesize, store and release neuronal growth factor (NGF) responsible for the growth and repair of nerve fibers [11-16]. The role of NGF in pathogenesis of HD is not known.

The purpose of our study was to estimate significance of morphometric MC study for diagnosis of Hirschsprung's disease.

\section{Materials and methods}

The study was performed on distal colon wall specimens harvested from three groups of patients. In group 3 (colon aganglionosis, $n=9$ ) and group 2 (constipation, normal ganglion cells, $n=6$ ) the full thickness samples were taken transtectally $1-2 \mathrm{~cm}$ above dental line. In group 1 (normal bowel function, normal ganglion cells, 
Table 1. Mean number of MC \pm standard deviation per $\mathrm{mm}^{2}$ of specimen in particular layers of the large bowel $\left(\right.$ cells $\left./ \mathrm{mm}^{2}\right)$

\begin{tabular}{|c|c|c|c|}
\hline & Control group & Constipations & Hirschsprung's disease \\
\hline Mucosa & $102.02 \pm 27.81$ & $102.74 \pm 15.6$ & $169.84 \pm 90.1$ \\
\hline Lamina propria & $127.05 \pm 29.49$ & $149.3 \pm 32.9$ & $221.61 \pm 155.15$ \\
\hline Submucosa & $97.16 \pm 40.34$ & $101.61 \pm 51.4$ & $125.54 \pm 34.28$ \\
\hline Muscle layer & $37.76 \pm 11.71$ & $23.17 \pm 15.99$ & $49.8 \pm 40.5$ \\
\hline Serosa & $70.95 \pm 30.46$ & $100 \pm 46.62$ & $69.57 \pm 40.3$ \\
\hline
\end{tabular}

Table 2. Mean measurements of the $\mathrm{MC} \pm$ standard deviation in submucosa.

\begin{tabular}{|l|c|c|}
\hline & Control group & Hirschsprung Disease \\
\hline Width $(\mu \mathrm{m})$ & $6 \pm 1.5$ & $7 \pm 1.5$ \\
\hline Length $(\mu \mathrm{m})$ & $6.2 \pm 1.6$ & $7.4 \pm 1.4$ \\
\hline Area $\left(\mu \mathrm{m}^{2}\right)$ & $38.2 \pm 17.3$ & $52.7 \pm 17.3$ \\
\hline Volume $\left(\mu \mathrm{m}^{3}\right)$ & $199 \pm 145$ & $309.4 \pm 158.4$ \\
\hline Elongation $(\mu \mathrm{m})$ & $1.5 \pm 0.3$ & $1.5 \pm 0.3$ \\
\hline
\end{tabular}

$\mathrm{n}=7$ ) the colon wall was sampled during surgical repairs performed because of anal atresia. The age of the patients ranged from 1 month to 12 years. The samples were examined according to the routine methods used to confirm or exclude diagnosis of Hirschsprung disease $[2,17]$. The diagnosis of HD was based on clinical symptoms, radiological findings, rectomanometric results. In every case the final diagnosis was confirmed histologicaly. None of the patients presented episodes of enterocolitis during or before biopsy. The patients with intestinal neuronal dysplasia were excluded.

The bowel wall specimens were fixed using a standard buffered $4 \%$ paraformaldehyde aqueous solution, executed through alcohol solutions due to standard technique protocol and embedded in paraffin. Staining was performed on $5 \mu \mathrm{m}$ paraffin sections which were first heated and then deparaffinised. Visualization of antibodies was obtained using LSAB technique according to manufactures protocol. Mast cells were localized in standard paraffin sections using immunohistochemical methods. Indirect biotyn-avidin-peroxidase technique with anti-human monoclonal antibodies was used. Visualization for the peroxidase was achieved by addition of 3,3'-diaminobenzydine as a substrate. Mast cells were localized using monoclonal antibody - Monoclonal Mouse Anti-Human Mast Cell Tryptase, Clone AA1, Isotype IgG1 (DakoCytomation, Code M 7052). In further stages we used antimouse antibody combined with biotin and peroxidase (Large Volume Dako LSAB ${ }^{\circledR}+$ Kit, Peroxidase, K 0690). Sections were counter-stained with haematoxylin.

The density of the mast cells was calculated using a light microscope Reichert- Jung with digital camera CCD Color Camera combined with IBM computer. LUCIA software was used as a morphometric tool. The MC were counted in five random fields in every layer of the bowel using light microscopy (x200). Morphometric MC measurements (length, width, area, volume, elongation) were performed in 50 random cells in submucosa and muscle layer in patients with HD and in control group. Test for the significance of differences between groups were performed using the KruskalWallis and U-Mann-Whitney tests.
Table 3. Mean measurements of the $\mathrm{MC} \pm$ standard deviation in muscle layer.

\begin{tabular}{|l|c|c|}
\hline & Control group & Hirschsprung Disease \\
\hline Width $(\mu \mathrm{m})$ & $6 \pm 1.5$ & $7 \pm 1.5$ \\
\hline Length $(\mu \mathrm{m})$ & $6.2 \pm 1.6$ & $7.4 \pm 1.4$ \\
\hline Area $\left(\mu \mathrm{m}^{2}\right)$ & $38.2 \pm 17.3$ & $52.7 \pm 17.3$ \\
\hline Volume $\left(\mu \mathrm{m}^{3}\right)$ & $199 \pm 145$ & $309.4 \pm 158.4$ \\
\hline Elongation $(\mu \mathrm{m})$ & $1.5 \pm 0.3$ & $1.5 \pm 0.3$ \\
\hline
\end{tabular}

\section{Results}

Significantly larger number of MC in mucosa and lamina propria $(\mathrm{p}<0.05)$ was found. In submucosa and muscle layer the difference was noticed but it was not statistically significant $(p>0.05 ; p<0.1)$. In the serosa there was no statistic difference in number of $\mathrm{MC}$ between groups $(p>0.1)$. Using statistic tests all measured morphometric parameters of the MC, except elongation, were significantly higher $(p<0.05)$ in patients with Hirschsprung disease.

We found, that MC were localized besides blood vessels and in contact with hypertrophic nerve trunks in aganglionic segment from HD (Fig. 1). In all diagnosed groups $\mathrm{MC}$ were localized in the greatest amount in mucosa and submucosa (Fig. 2, 3).

Mast cells in patients with Hirschsprung's disease, in light microscopy, were morphologicaly similar to cells in control group. In all cases they presented morphology typical for MC and they can be described as "fried eggs". We also found out, that muscle layer in control group had smaller number of MC in comparison to muscle layer of patients with HD. MC were localized in muscle layer solitary or in groups (Fig. 4, 5). Results are summarized in Table 1, 2 and 3.

\section{Discussion}

MC have been found to be involved in many reactions in human body. They can be found in many tissues. A large number of $\mathrm{MC}$ is localized on the border of our 

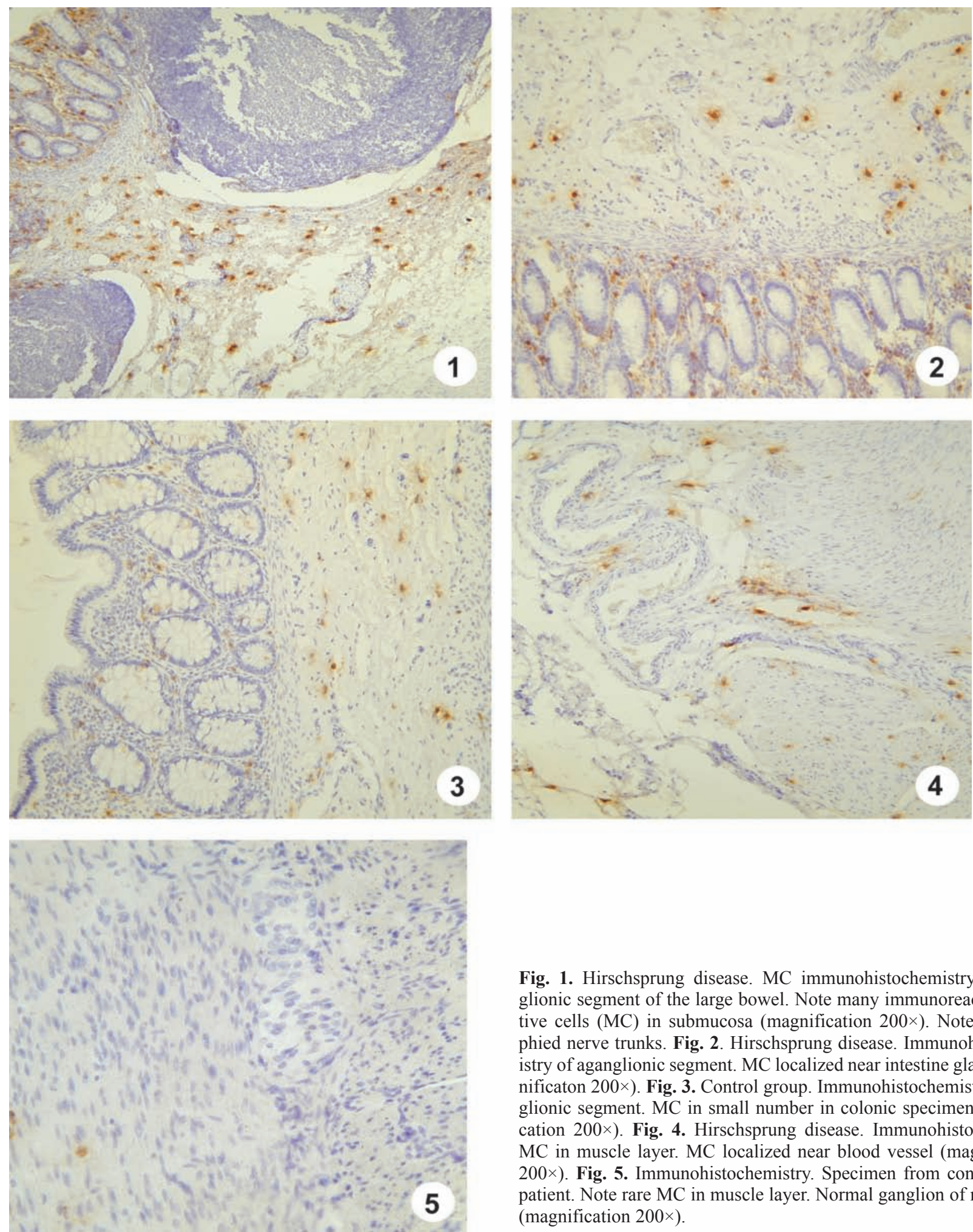

Fig. 1. Hirschsprung disease. MC immunohistochemistry of aganglionic segment of the large bowel. Note many immunoreactive-positive cells (MC) in submucosa (magnification 200×). Note hypertrophied nerve trunks. Fig. 2. Hirschsprung disease. Immunohistochemistry of aganglionic segment. MC localized near intestine glands (magnificaton 200×). Fig. 3. Control group. Immunohistochemistry of ganglionic segment. MC in small number in colonic specimen (magnification 200×). Fig. 4. Hirschsprung disease. Immunohistochemistry. $\mathrm{MC}$ in muscle layer. MC localized near blood vessel (magnification $200 \times$ ). Fig. 5. Immunohistochemistry. Specimen from control group patient. Note rare MC in muscle layer. Normal ganglion of nerve cells (magnification 200×).

body and external environment, especially in skin, lungs and digestive tract. They are also localized near nerve fibers and blood vessels. Releasing numerous products contained in their cytoplasmatic granules, $\mathrm{MC}$ regulate and orchestrate many different physiological processes. MC, through neuronal growth factor
(NGF) influence also on nervous system $[6,11,12,15,16,18-20]$. Other authors $[13,14]$ showed elevated content of NGF and increased number of MC in the aganglionic colon wall in Hirschsprung's disease in children. There are just three papers in literature about mast cells in Hirschsprung's disease. 
It was found in the present study, that morprometric parameters in aganglionic segment are increased comparing with normally inervated colon. Enlargement of $\mathrm{MC}$ is an indirect proof of MC activation in HD. It is hard to say why these cells are activated in aganglionic segment. Morphology of MC in both groups is the same so the biological role should be also similar. In physiological conditions they are responsible especially for inflammation but among many other functions of mast cell they probably play role in reinervation process. It is very interesting that in aganglionic segment of the bowel wall all measured parameters of the MC are significantly higher compared with control group. During activation process MC produce, cumulate and release great amount biologically active substances in their cytoplasmatic granules. Probably some of these substances can effect on defected nervous system.

Furthermore, we also noticed that some of the colon mast cells in HD were located near or they were even attached to the hypertrophic nerve trunks. This is another proof of connection between defected nervous system in aganglionic bowel and mast cells. We don't know how close this connection is but MC probably affect on hyperthrophied nerve trunks. We couldn't observe this in normal bowel wall with regular ganglions.

We also analyze density of the mast cells in each layer of the large bowel. Other authors analyzed distribution of MC along and across of digestive tract $[5,9]$. We revealed that there is a greater number of MC in mucosa, lamina propria, submucosa and muscle layer of the large bowel in patients with Hirschsprung's disease. There was no difference in density of the MC in serosa. This is quite similar with findings of other authors $[13,14]$. This also proves that higher number of mast cells is probably caused by defect of the nervous system.

Mobilization and activation of the mast cells can be directed to repair defective nervous system. Obviously, MC repairing reaction is not effective in restoring nervous system function of the gut. Although in HD we have probably activated MC and they are probably producing larger amount of NGF we still have defective nervous system and all symptoms related with this. So this is certain that NGF it is not single substance responsible for repairing $[18,21,22]$.

We still don't know exact role of $\mathrm{MC}$ in Hirschsprung's disease. The data are not enough to support the fact that $\mathrm{MC}$ are a part of repairing system of defective nervous system of the bowel in HD, but this data suggest that they play an important role in pathogenesis of HD.

\section{References}

[1] Gershon M and Ratcliffe E. Developmental biology of the enteric nervous system: pathogenesis of Hirschsprung's disease and other congenital dysmotylities. Seminars in pediatric surgery. 2004;13:224-235.
[2] Meier-Ruge W, Morphological diagnosis of Hirschsprung's disease. Hirschsprung's disease, ed. Holschneider M and Puri P. 1982, New York: Thieme-Stratton. 252-265.

[3] Puri P and Shinkai T. Pathogenesis of Hirschsprung's disease and its variants: recent progress. Seminars in Pediatric Surgery 2004;13:18-24.

[4] Swenson O. Hirschsprung's disease: a review. Pediatrics. 2002;109:914-918.

[ 5] Barczyk M, Dębek W, Chyczewski L. Mast cells in the gastrointestinal tract. Annales Academiae Medicae Bialostociensis. 1995;40:36-57.

[ 6] Buys J and Ruitenberg E. Mucosal mast cells: origin, kinetics and functions. Wiad Parazytol. 1986;32:209-231.

[7] Enerback L. Mast cell in rat gastrointestinal mucosa. Acta Pathol Microbiol Scand. 1966;66:313-322.

[ 8] Enerback L, Mast cell heterogeneity: the evolution of the concept of a specific mucosal mast cells. Mast cell differentiation and heterogeneity, ed. Befus A, Bienenstock J, and Denburg J. 1986, New York: Raven Press. 1-21.

[ 9] Furgal A and Litwin J. Distribution of mast cells along and across successive segments of the rat digestive tract: a quantitative study. Folia Histochemica et Cytobiologica. 1998;36:19-27.

[10] Guy-Grand D, et al. Gut mucosal mast cells. Origin, traffic and differentiation. $J$ Exp Med. 1984;160:12-28.

[11] Bonini S, et al. Nerve growth factor: neurotropin or cytokine? Arch. Of Allergy and Immunology. 2003;131:80-84.

[12] Caughey G. Serine proteases of mast cell and leukocyte granules. Americam Journal of Respiratory and Critical Care Medicine. 1994;150:138-142.

[13] Demirbilek S, Ozardali H, Adym G. Mast-cell distribution and colonic mucin composition in Hirschsprung's disease and intestinal neuronal dysplasia. Pediatr. Surg. Int. 2001;17:136-139.

[14] Kobayashi H, et al. Mast cells and gut nerve development: implications for Hirschsprung's disease and intestinal neuronal dysplasia. J Ped Surg. 1999;34:543-548.[18] Dines $\mathrm{K}$ and Powell $\mathrm{H}$. Mast cell interactions with nervous system: relationship to mechanisms of disease. Journal of neuropathology and experimental neurology. 1997;56:627-640.

[15] Levi-Schaffer F, et al. Nerve growth factor is produced by human fibroblasts and modulates them: role of Nerve growth factor in tissue repair. International archives of allergy and immunology. 2001;124:279-281.

[16] Stassen M, et al. Mast cells and inflamation. Archivum Immunologiae et therapiae experimentalis. 2002;50:179-185.

[17] Heitz P and Komminoth P. Biopsy diagnosis of Hirschsprung's disease and related disorders. Curr Top Pathol. 1990;59:257-275.

[18] Dines K and Powell H. Mast cell interactions with nervous system: relationship to mechanisms of disease. Journal of neuropathology and experimental neurology. 1997;56:627-640.

[19] Dvorak A, et al. Human gut mucosal mast cells: ultrastructural observations and anatomic variation in mast cell-nerve associations in vivo. Int. Arch. Allergy Appl Immunol. 1992;98:158-168.

[20] Leon A, et al. Mast cells synthesize, store and release nerve growth factor. Proc Natl Acad Sci USA. 1994;91:3739-3743.

[21] Blennerhassett M, Tomioka M, and Bienenstock J. Formation of contacts between mast cells and sympathetic neurons in vitro. Cell Tissue Res. 1991;265:121-128.

[22] Coskun M, Sindel M, Elpek G. Mast cell density, neuronal hypertrophy and nerve growth factor expression in patients with acute appendicitis. Folia Morphol. 2002;61:237-243.

[23] Dvorak A. New aspects of mast cell biology. Int. arch. Allerg. Immunol. 1997;114:1-9.

Submitted: 2 April, 2007 Accepted after reviews: 31 August, 2008 